Med Klin Intensivmed Notfmed 2020 • 115:6-7 https://doi.org/10.1007/s00063-019-00649-z

(c) Springer Medizin Verlag GmbH, ein Teil von Springer Nature 2020

„Stationäre Behandlung - Der alte $\mathrm{Pa}$ tient wird zum Normalfall" war der Titel eines Artikels von Birgit Hibbeler im Deutschen Ärzteblatt 2013 [1], in dem einmal mehr deutlich wurde „alte Menschen sind vulnerabler als junge“. Zwischenzeitlich ist der „Normalfall“ längst eingetreten. Die Zahl von hochbetagten Patienten über 85 Jahren hat im Krankenhaus kontinuierlich von 2000-2015 um $24,2 \%$ zugenommen [2]. Hieraus erwächst auch der Bedarf für eine perioperative Altersmedizin, der kürzlich mit konsentierten Empfehlungen von einer interdisziplinären Arbeitsgruppe um die Gerontoanästhesisten Cynthia Olotu und Rainer Kiefmann formuliert wurde [3]. Dies gilt nicht nur für die Vorbereitung elektiver Eingriffe, sondern gerade für das besonders kurze Zeitfenster in der Notfallsituation. So müssen die funktionellen Altersveränderungen und Stoffwechselbesonderheiten des alten Menschen besondere Berücksichtigung erfahren. Da, wo Geriater nicht sofort verfügbar sind, setzt dies bei allen im therapeutischen Team ein besonderes Wissen und klinische Erfahrung voraus. Notwendig sind angepasste strukturierte Prozesse schon in der Notaufnahme, die Angehörige und Begleiter sowie das Vorliegen einer Patientenverfügung mit einbeziehen.

In diesem Rahmen möchte diese interdisziplinäre Ausgabe der Zeitschrift Medizinische Klinik - Intensivmedizin und Notfallmedizin für den geriatrischen Notfallpatienten wichtige Aspekte von der Notaufnahme über die Operation bis hin zur Intensivstation aufgreifen. Die Autoren haben aus der Zusammenstellung der Literatur und der eigenen Erfahrung jeweils praktische Handlungsempfehlungen entwickelt.

\author{
A. Weimann' $\cdot$ U. Müller-Werdan ${ }^{2}$ \\ ${ }^{1}$ Klinik für Allgemein-, Viszeral- und Onkologische Chirurgie, Klinikum St. Georg, Leipzig, Deutschland \\ ${ }^{2}$ Klinik für Geriatrie und Altersmedizin, Evangelisches Geriatriezentrum Berlin, Charité - \\ Universitätsmedizin Berlin, Berlin, Deutschland
}

\title{
Geriatrische Notfälle
}

Zunächst geben die Notfallmediziner Michael Groening und Petra Wilke Empfehlungen für die Notaufnahme geriatrischer Patienten, deren Triage sowie geeignete Screeninginstrumente zur Erfassung der Funktionalität. Die adäquate Betreuung gerade dementer Patienten in der Notaufnahme wird besonders dargestellt. Thematisiert werden auch die ethischen Aspekte mit der vom Patienten gewünschten Behandlungsintensität.

Die Gerontoanästhesistin Cynthia Olotu geht auf die besonderen Aspekte der Notfallanästhesie beim alten Menschen ein. Ein Fokus liegt auf der Erkennung und Vermeidung des prognostisch ungünstigen Delirs in Abgrenzung zur Demenz.

Der Chirurg Cornelius van Beekum und Kollegen belegen mit eigenen Ergebnissen die Unterschiede des mechanischen Ileus beim alten Menschen im Vergleich mit jüngeren Patienten sowie die Indikation zur Notfalloperation. Aus der Erfahrung der Autoren resultiert ein Algorithmus zum Vorgehen schon in der Notaufnahme.

Schließlich gibt der Nephrologe Carsten Hafer eine aktuelle Übersicht zu der beim geriatrischen Patienten auf der Intensivstation häufigen Hyponatriämie und ihrer metabolisch angepassten Therapie mit einem Workflow.

Mit großem Dank an die Autoren und in der Hoffnung auf Interesse und Rezeption

Ursula Müller-Werdan und Arved Weimann

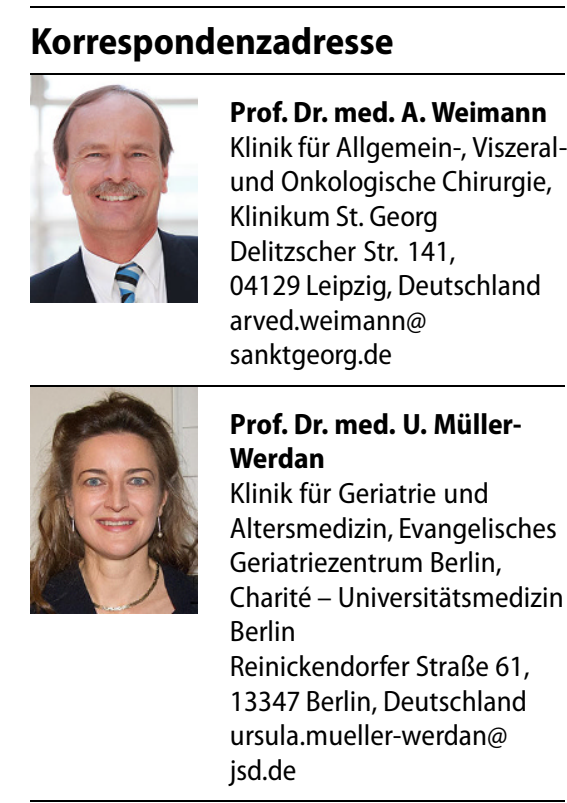

Interessenkonflikt. A. Weimann und U. Müller-Werdan geben an, dass kein Interessenkonflikt besteht.

\section{Literatur}

1. Hibbeler B (2013) Stationäre Behandlung - Der alte Patient wird zum Normalfall. Dtsch Arztebl 110(21):A-1036-C-898

2. Schelhase T(2018) Statistische Krankenhausdaten Diagnosedaten der Krankenhauspatienten 2015. In: Klauber J, Geraedts M, Friedrich J, Wasem J (Hrsg) Krankenhaus-Report 2018, S 381 (Tab. 20-1)

3. Olotu C, Weimann A, Bahrs C, Schwenk W, Scherer M, Kiefmann R (2019) Bedarf für eine perioperative Altersmedizin - eine interdisziplinäre Aufgabe. Dtsch Arztebl Int 2019(116):63-69 
Hier steht eine Anzeige.

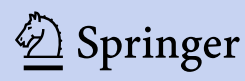

